\title{
Journal of Sports Injuries and Medicine
}

\section{Eccentric Loading Rehabilitation of Patient with Severe Post- Operative Muscle Atrophy}

\author{
Robert Donatelli, Zara Q Colthart, Randa Bascharon* \\ Orthopedic Care Medical Legal Consulting, orthopedic surgeon in Las Vegas, USA
}

"Corresponding author: Randa Bascharon, Orthopedic Care Medical Legal Consulting, orthopedic surgeon in Las Vegas, USA, Tel: +1 7029477790; E-mail: Rbascharon@vegasortho.com

Citation: Donatelli R, Colthart ZQ, Bascharon R (2017) Eccentric Loading Rehabilitation of Patient with Severe Post-Operative Muscle Atrophy. Sports Injr Med JSIMD 2017: 107. DOI: 10.29011/ JSIMD-107. 100007. DOI: 10.29011/ JSIMD-107. 100007

Received Date: 5 March, 2017; Accepted Date: 20 March, 2017; Published Date: 27 March, 2017

\begin{abstract}
This case is a demonstration of the benefits of utilizing eccentric exercises early in the rehabilitation of post-op left knee arthroscopy for partial lateral meniscectomy, excision of the plica and chondroplasty with micro fracture. Secondary to the injury and then post-op the patient demonstrated severe muscle atrophy and post-op pain. Trauma to a joint causing joint effusion followed by disuse, resulted in loss of strength, stabilization and function. It is important to facilitate and promote muscle strengthening early in the rehabilitation. There are various exercises that utilize three different types of muscle actions concentric, eccentric or isometric contractions. Eccentric loading, especially in rehabilitation, is the most efficacious method of increasing strength gains while minimizing the unfavorable effects caused by compression forces into the joint, especially postop surgeries that involve intra-articular repair. The purpose of this patient case is to demonstrate the effectiveness of eccentric loading in the recovery of muscle strength in the lower limb, improvement in function and to suggest its implementation as an important part of rehabilitation programs.
\end{abstract}

\section{Introduction/Background}

Mechanical tension, muscle damage and metabolic stress are mechanisms by which exercise-induced hypertrophy occurs [1]. Mechanical tension, which can occur through force production in resistance training, induces mechanical overload on the muscle, disrupting its myofibrils and facilitation of satellite cells activation $[1,2]$. This disruption stimulates satellite cells to promote muscle growth by multiplying, forming new myofibrils, donating nuclei to muscle fibers, and co-expressing myogenic regulatory factors that assist in the reparation, regeneration and growth of muscle tissue1. Resistance training also creates micro tears within the muscle, and the body responds to the damage by sending neutronphils to the area of trauma. The result is an influx of macrophages and lymphocytes that eventually act to increase satellite cell proliferation 1 and therefore, muscle growth. Finally, high-intensity exercise utilizes anaerobic glycolysis for ATP production, thereby creating an accumulation of metabolites such as lactate and hydrogen ions. This build-up creates a hypertrophic response through hormones, growth factors; cell-swelling and free-radicals [1].The resulting acidic environment may also stimulate sympathetic nerves to further increase muscle hypertrophy [1].
Muscle contractions can be either concentric or eccentric. During a concentric contraction, the muscle shortens whereas during an eccentric contraction, the muscle lengthens [3]. Eccentric muscle lengthening combined with overload increases passive muscular tension of the contractile units in the muscle $[1,2]$. Because passive muscular tension is much higher in eccentric contractions as opposed to concentric contractions, the contractile units will undergo greater sub cellular damage and therefore demonstrate a more dramatic hypertrophic response from eccentric work1, 2. Many studies have proven that exercise involving solely eccentric contractions will result in an increase in the number of sarcomeres in series [1,4] and parallel [2], while sarcomere length decreases with exclusively concentric exercise $[1,2]$. The addition of sarcomeres in series has proven Effective in Curbing ExerciseInduced Muscle Damage (EIMD). EIMD occurs after unaccustomed exercise and causes a host of negative events, among which are swelling, soreness and reduced force production; therefore, the adherence to and success of rehabilitation programs is compromised with EIMD [5]. EIMD is less severe following eccentric work because of increased muscle compliance resulting from the addition of sarcomeres in series [4]. Conversely, concentric exer- 


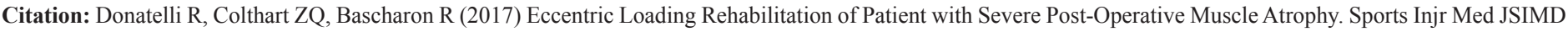
2017: 107.

cise increases the likelihood of EIMD because of the reduction of sarcomeres in series [4].

Eccentric exercise becomes even more beneficial when utilized for muscle rehabilitation. When a muscle is injured, a hematoma is formed at the site of injury and inflammatory cells and growth factors flood the area [6]. Some of the growth factors will activate satellite cells, eventually proliferating into myoblasts that attach to and repair the damaged myofibers [6]. Scar tissue is then formed with the purpose of reinforcing the muscle during contractions, but is considerably weaker and therefore presents a concern for re-injury [6]. If the injured muscle is not exercised, however, the healing process will be stunted by over-deposition of scar tissue. Therefore, exercise that does not exacerbate the injury is essential to the healing process. Eccentric exercise consumes less oxygen and energy while producing a greater amount of force [7] and stimulates hypertrophy post-injury [2]. The stimulus of great muscle mass may be the more prudent option for rehabilitation.

\section{Case Study}

A 26 year old competitive male power lifter was sent to physical therapy with a physician diagnosis of post-operative osteochondral defect, lateral meniscus tear and impinging plica of the left knee. The patient complained of left knee pain and stiffness, particularly with extension. He claimed the first incident of knee injury occurred at a power lifting competition 1 year prior from hyperextension of the knee during a dead lift. The patient did not seek treatment immediately, but saw a physician once the pain worsened following a different competition. The physician performed a left knee arthroscopy, partial lateral meniscectomy, excision of the plica and chondroplasty with micro fracture. The patient was instructed to wait 8-10 weeks before bearing weight on the left leg. The patient began physical therapy approximately 4 weeks post-surgery.

Bilateral weakness of the posterior hip muscles, limited left knee extension (by $15^{\circ}$ active) and left side weakness of the quads and hamstrings were found in the initial evaluation. The goals of physical therapy were to increase strength and ROM of the left knee through eccentric strength training and manual therapy.

\section{Intervention}

The treatment began with exercise on the stationary bike as well as 3 sessions of manual therapy. The goal of both was to reestablish ROM in the knee in order to gain full knee extension. Once full ROM was achieved, the patient began posterior lateral exercises using a pulley for resistance to increase hip strength. After hip strength increased, the focus of therapy was eccentric strength training using a computerized machine called "Eccentron", (by BTE) a device that only offers eccentric resistance. Eccentric loading was implemented approximately 4 weeks post-surgery and continued for 15 weeks. The subject performed 12 strides on the machine at maximal effort. The machine discarded the greatest force value and halved the second greatest force value of the weaker leg to determine the recommended resistance. Therefore, the subject was working at $50 \%$ of sub-maximal force of the weaker leg. A maximum and minimum range of force production was calculated to create a target zone. The subject had to remain within the target zone for each session in order to progress to the next level of dosing. The workout increased in 5 minute increments for the first 3 sessions.

After the first 3 sessions, the duration of the workout increased in smaller increments until it reached the maximum of 30 minutes. Re-testing was implemented every 3 sessions in order to adjust the resistance to match the subject's improvements in strength.

\section{Results}

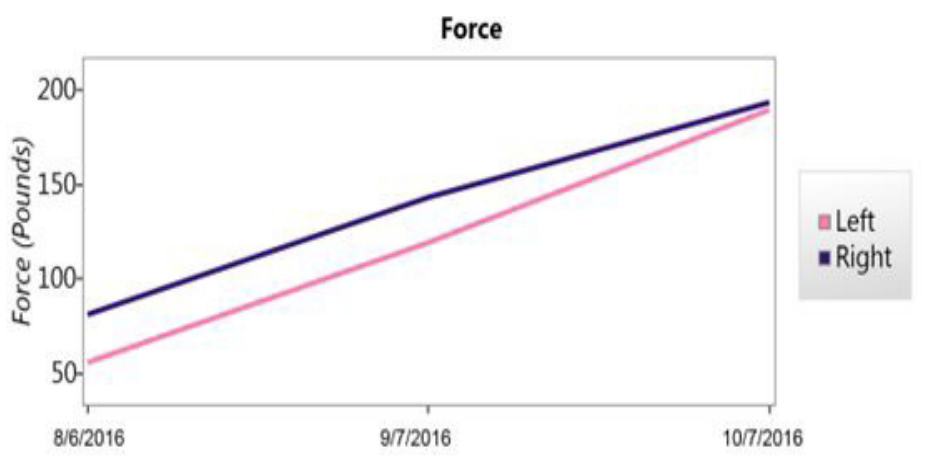

Figure 1: Changes in force production over a two-month period.

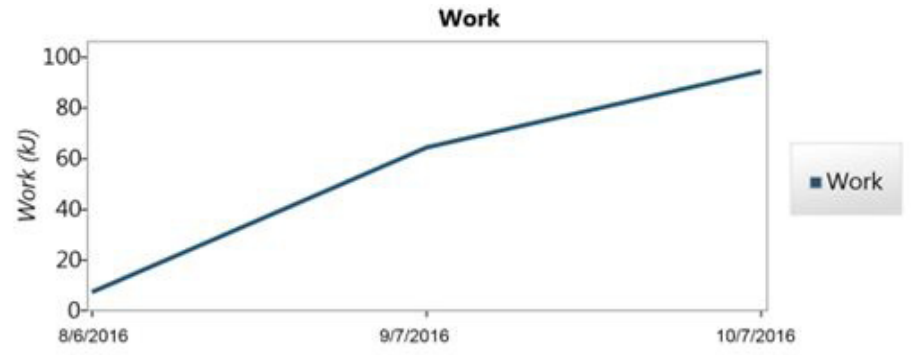

Figure 2: Changes in work over a two-month period.

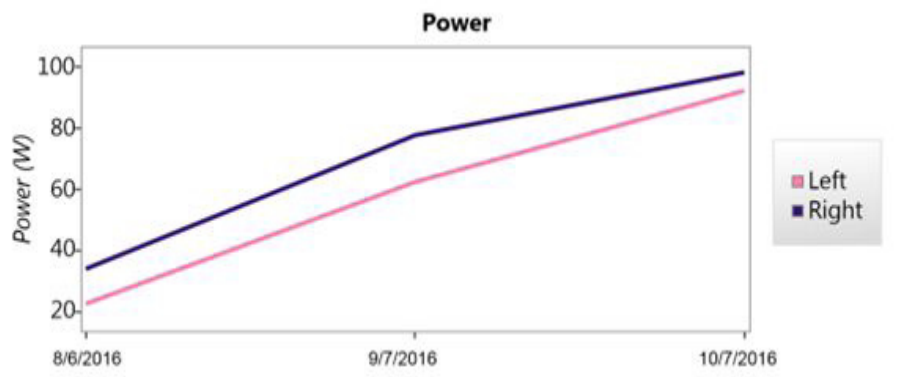

Figure 3: Changes in power over a two-month period. 


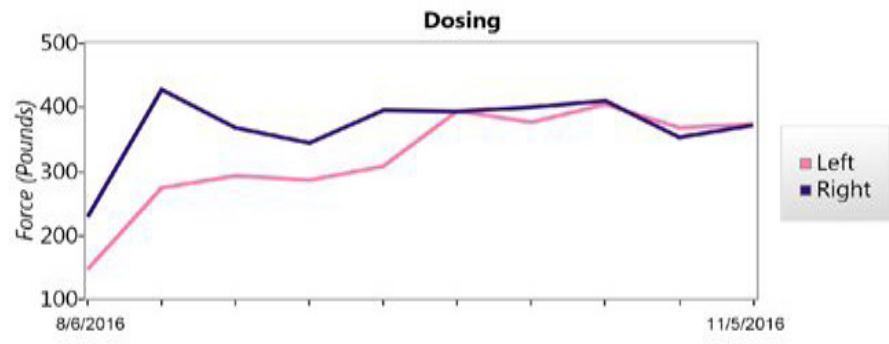

Figure 4: Changes in dosing over a two-month period.

\begin{tabular}{|l|c|c|c|c|}
\hline $\begin{array}{c}\text { First / Last Date } \\
\text { Comparisons }\end{array}$ & L & Change & R & Change \\
\hline Avg Force & $\begin{array}{c}\mathbf{5 4 . 9 7 / 1 8 8 . 2} \\
\text { Pounds }\end{array}$ & $242 \%$ & $\begin{array}{c}80.33 / 192.1 \\
\text { Pounds }\end{array}$ & $139 \%$ \\
\hline Peak Force & $\begin{array}{c}76.58 / 236.0 \\
\text { Pounds }\end{array}$ & $208 \%$ & $\begin{array}{c}193.5 / 258.9 \\
\text { Pounds }\end{array}$ & $33.8 \%$ \\
\hline Work & \multicolumn{2}{|c|}{$7.000 / 94.00 \mathrm{~kJ}$} & \multicolumn{2}{|c|}{$1243 \%$} \\
\hline Power & $\mathbf{2 1 . 8 4 / 9 1 . 4 7} \mathrm{W}$ & $\mathbf{3 1 9} \%$ & $\mathbf{3 3 . 2 8} / \mathbf{9 7 . 4 5} \mathrm{W}$ & $193 \%$ \\
\hline \% on Target & $\mathbf{6 9 . 1 1 / 1 0 0 . 0}$ & $\mathbf{4 4 . 7} \%$ & $\mathbf{8 5 . 5 0} / \mathbf{9 9 . 7 7}$ & $16.7 \%$ \\
\hline Dosing & $\begin{array}{c}145.6 / 371.6 \\
\text { Pounds }\end{array}$ & $155 \%$ & $\begin{array}{c}227.3 / 369.7 \\
\text { Pounds }\end{array}$ & $\mathbf{6 2 . 6} \%$ \\
\hline
\end{tabular}

\begin{tabular}{|l|c|c|}
\hline Average Speed & $\mathbf{2 2 . 6 0} / \mathbf{2 7 . 6 0} \mathrm{rpm}$ & $\mathbf{2 2 . 1} \%$ \\
\hline Session Duration & $\mathbf{3 0 0 . 0} / \mathbf{1 2 0 0}$ Seconds & $\mathbf{3 0 0} \%$ \\
\hline
\end{tabular}

Figure 5: Data summary from Eccentron over a two-month period.

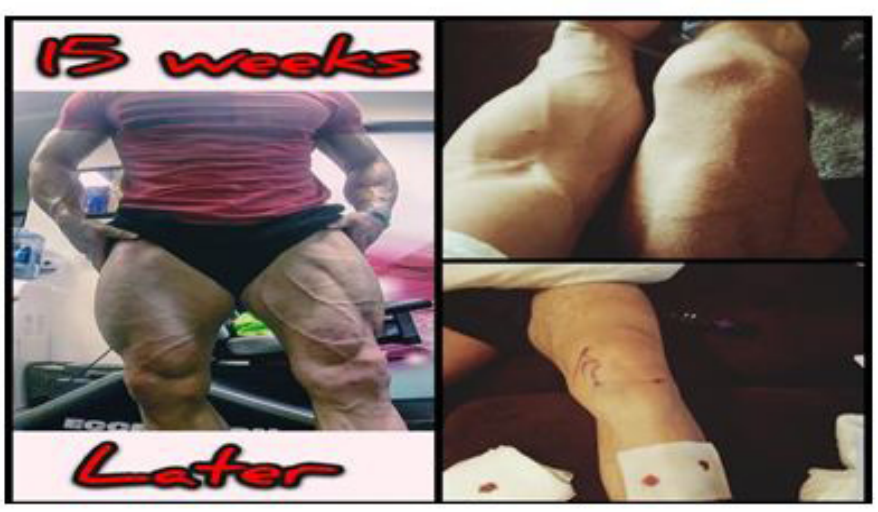

Figure 6: (right) Left quadriceps 2 weeks post-op, before therapy (disuse atrophy noted) (left) Quadriceps after 15 weeks of rehabilitation with use of the Eccentron (30 minutes, $3 \mathrm{x}$ weekly).

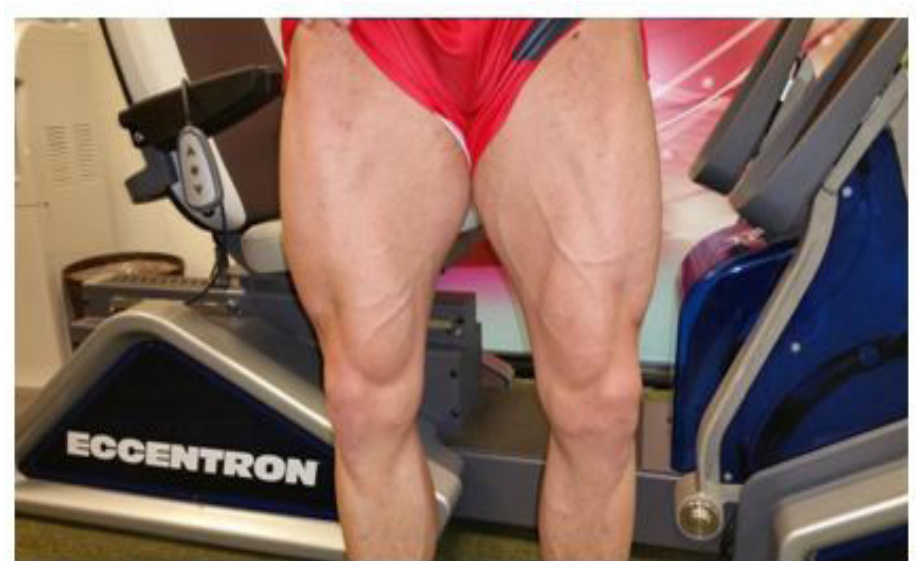

Figure 7: Quadriceps after 15 weeks of rehabilitation with use of the Eccentron (30 minutes, $3 \mathrm{x}$ weekly).

\section{Discussion}

The rapid increase in lower-extremity strength found in this case study demonstrates the efficacy of utilizing eccentric loading for rehabilitation. Valero et al [8] have found that eccentric work is an effective promoter of repair and recovery because of its ability to stimulate the release of mesenchymal stem cells within the muscle. Additionally, following eccentric exercise, Valero et al8 noted an increase in a [7] integrin which is useful in overcoming muscle atrophy. Eccentric loading may also be a more viable option in rehabilitation settings because of the protective role that eccentric muscle contractions provide, such as deceleration. Eccentric isokinetic testing is used in determining return to play post-injury and therefore proves a useful tool in the prediction of re-injury [9]. Therefore, the strengthening of deceleration muscles through eccentric work is functional for both the athlete and non-athlete alike, and can be applied in a multitude of rehabilitation settings.

The present study has weaknesses simply because it is a single case. Future research could be a comparative study, designed to have a control and experimental group. Eccentric exercises can be done using traditional exercises with free weights and body weight. A research question for future studies could be, are traditional exercises just as effective as exercising on the Eccentron in building muscle strength and muscle hypertrophy? 
Citation: Donatelli R, Colthart ZQ, Bascharon R (2017) Eccentric Loading Rehabilitation of Patient with Severe Post-Operative Muscle Atrophy. Sports Injr Med JSIMD 2017: 107 .

\section{Conclusion}

Muscle injury is often caused by and/or causes a reduction in strength. Therefore, muscle hypertrophy is a common goal in rehabilitation. Eccentric loading overloads the muscle in a manner that intensifies hypertrophy without inducing the excessive damage to muscle cells that often occurs with concentric loading. Eccentric loading is a viable option for rehabilitation in terms of speed and quantity of strength gains, as well as safety and functionality of movement. For this reason, eccentric work should be considered in the rehabilitation of injuries.

\section{References}

1. Schoenfeld J (2010) The mechanisms of muscle hypertrophy and their application to resistance training. Journal of Strength and Conditioning Research 24: 2857-2872.

2. Hedayatpour N, Falla D (2015) Physiological and neural adaptations to eccentric exercise: mechanisms and considerations for training. Biomed Research International, 2015.

3. Padulo J, Laffaye G, Chamari K (2013) Concentric and eccentric: muscle contraction or exercise? Journal of Sports Science and Medicine 12: 608-609.
4. Gleeson N, Eston R, Marginson V, Mchugh M, Bird S (2003) Effects of prior concentric training on eccentric exercise induced muscle damage. British Journal of Sports Medicine, 37: 119-125.

5. Howatson G, Van Someren KA (2008) The prevention and treatment of exercise-induced muscle damage. National Center for Biotechnology Information, 38: 483-503.

6. Baoge L, Van Den Steen E, Rimbaut S, Philips N, Witvrouw E, et al. (2012) Treatment of skeletal muscle injury: a review. International Scholarly Research Notices, 2012.

7. Lorenz D, Reiman M (2011) The role and implementation of eccentric training in athletic rehabilitation: tendinopathy, hamstring strains, and acl reconstruction. International Journal of Sports Physical Therapy, 6: $27-44$.

8. Valero M, Huntsman H, Liu, Zou K, Boppart, M (2012) Eccentric exercise facilitates mesenchymal stem cell appearance in skeletal muscle. PLoS One, 7(1).

9. Croisier J, Croisier J, Kaux J, Crielaard J, Forthomme B (2015) The relevance of eccentric isokinetic intervention. Annals of Physical and Rehabilitation Medicine 58: 160. 